\title{
Cardiac MRI use in the diagnosis of acute infectious myocarditis
}

\author{
Heather Wheat, Jamal Hajjari, Claire Sullivan \\ University Hospitals Cleveland Medical Center, Cleveland, Ohio, United States
}

Received: January 26, 2019

Accepted: March 24, 2019

Online Published: April 23, 2019

DOI: $10.5430 /$ crim.v6n2p11

URL: https://doi.org/10.5430/crim.v6n2p11

\begin{abstract}
Viral myocarditis is a prevalent disease amongst endemic nations. With increased opportunity of travel, and warmer climates for arthropod-vectors to spread, infectious myocarditis incidence is likely to rise as well. Acute manifestations of myocarditis include fevers, generalized weakness, myalgias, and arthralgias. While death is rare, long-term morbidity related to acute complications plus chronic cardiac structural changes can occur. No current guidelines for long-term myocarditis follow-up and designated treatment strategies exist, and future work in these areas is required.
\end{abstract}

Key Words: Myocarditis, Infectious, Cardiac MRI

\section{INTRODUCTION}

Infective myocarditis is a relatively common pathology worldwide, making up the majority of the approximately 1.5 million cases of myocarditis diagnosed each year. ${ }^{[1]}$ Infectious myocarditis is likely underdiagnosed, as proper diagnosis requires endomyocardial biopsy (EMB) that is often not performed due to limited clinical significance and treatment focused on supportive measures. ${ }^{[1,2]}$ While infectious etiologies can include bacteria, fungi, and helminths, the most common cause is viral, most of which include the picornavirus, herpes virus, and parvovirus families. ${ }^{[1,2]}$ Many infectious etiologies are spread by arthropods, including Dengue fever and West Nile, which are often endemic to tropical regions. Of late, many endemic infections have spread to more arid regions with increased travel. Furthermore, as global temperatures rise, arthropod-driven infections are expected to rise, posing a public health problem and increasing the risk for spread to non-endemic regions.
Clinical features of viral myocarditis can involve variable symptoms, ranging from fevers, myalgias, arthralgias, and arrhythmias, to fulminant heart failure with cardiogenic shock. ${ }^{[3,4]}$ Patients can present in the acute stages of infection, or can present several years later with signs of myocardial fibrosis and cardiomyopathy. ${ }^{[1]}$ While EMB is the diagnostic standard for myocarditis, biopsy is not only invasive, but in cases of suspected viral myocarditis, is not typically indicated as management is largely supportive, with resolution of symptoms within weeks. ${ }^{[1,2]}$ This case presents a 47-year-old male with the development of suspected viral myocarditis after vacationing in the US Virgin Islands.

\section{Case presentation}

A 47-year-old Caucasian male with a past medical history significant for gout originally presented to his outpatient primary care physician in Ohio four days after returning from a week-long trip to St. Croix in the US Virgin Islands with his wife, who was asymptomatic. He complained of daily

\footnotetext{
*Correspondence: Heather Wheat; Email: Heather.Wheat@UHHospitals.org; Address: University Hospitals Cleveland Medical Center, Cleveland, Ohio, United States.
} 
fevers measuring $38.2^{\circ} \mathrm{C}-39.4^{\circ} \mathrm{C}$ minimally responsive to Tylenol, episodes of diaphoresis, diffuse muscle aches, generalized malaise, large joint pains, and decreased appetite. Initial vitals and labs are provided in Table 1. After labs were drawn for Dengue, typhus, hepatitis panel, and West Nile, the patient was sent home and returned two days later to the Emergency Department at University Hospitals Cleveland Medical Center with persistent fevers following an episode of chest tightness that improved upon sitting, orthopnea, and cough with blood-tinged sputum. The patient suffered several bug bites during his trip, with resolving bites located on his bilateral feet. He developed a $10 \mathrm{~cm} \times 10 \mathrm{~cm}$, pruritic patch of erythema without central clearing one day after arriving in St. Croix, without inciting injury, skin breakdown, or a known bug bite at the site. The following day, the rash blistered with yellow, purulent drainage before resolving after 5 days with the use of a topical spray. He denied any vaccinations prior to travel, drank only from bottled water, and did not consume street-food. During his stay, he did not hike, but did pet one wild cat.

Table 1. Laboratory tests

\begin{tabular}{llll}
\hline Laboratory Tests & $\begin{array}{l}\text { Primary } \\
\text { Care Office }\end{array}$ & $\begin{array}{l}\text { Emergency } \\
\text { Department }\end{array}$ & $\begin{array}{l}\text { Normal } \\
\text { range }\end{array}$ \\
\hline Leukocytes $\left(\times 10^{9}\right)$ & 13.2 & 10.8 & $4.4-11.3$ \\
Hemoglobin $(\mathrm{g} / \mathrm{dl})$ & 16.5 & 16.6 & $13.5-17.5$ \\
Hematocrit $(\%)$ & 49.5 & 46.8 & $41.0-52.0$ \\
Platelets $\left(\times 10^{9}\right)$ & 224 & 229 & $150-450$ \\
Cr $(\mathrm{mg} / \mathrm{dl})$ & 0.86 & 0.77 & $0.6-1.3$ \\
Troponin $(\mathrm{ng} / \mathrm{ml})$ & & 12.47 & $0.00-0.03$ \\
Lactate $(\mathrm{mmol} / \mathrm{L})$ & & 2.5 & $0.4-2.0$ \\
CRP $(\mathrm{mg} / \mathrm{dl})$ & & 11.11 & $<1.00$ \\
\hline
\end{tabular}

Vitals and labs drawn in the ED are presented in Table 1. On clinical exam, the patient was diaphoretic, had mild posterior pharyngeal erythema, and diffuse bilateral crackles at the pulmonic bases with decreased basilar fremitus and no egophony. Several resolving bug bites were noted on bilateral feet without edema or cyanosis. A 12-lead ECG detected sinus tachycardia, PACs, left-axis deviation, and non-specific ST/T wave changes. Initial CXR was negative for acute process and troponin was 12.47. The patient was loaded with ticagrelor $180 \mathrm{mg}$ and started on a heparin drip. Additional labs were drawn for blood cultures, urine culture, IgM, adenovirus, coxsackie virus, parvovirus B19, chikungunya, Rocky Mountain Spotted fever, HIV, influenza, and zika, and the patient was hospitalized.

On the first night, the patient spiked a fever to $39.2^{\circ} \mathrm{C}$. Cardiology was consulted on the second day of hospitalization and recommended adding ASA $81 \mathrm{mg}$ daily, metoprolol 12.5 $\mathrm{mg}$ q6h due to high risk for arrhythmia, and atorvastatin 40 mg. A transthoracic echocardiogram was performed, which demonstrated a left ventricular ejection fraction of 45\%-50\% and no significant valvular pathology. Given high clinical concern for myocarditis, a cardiac MRI was performed and was significant for areas of scattered mid-myocardial and epicardial delayed enhancement of the anterior, septum, and inferior walls concerning for myocarditis (see Figures 1 and 2). Evaluation of the patient's coronary arteries prior to pursuing cardiac MRI was not completed as the clinical suspicion for coronary artery disease was low given his limited ASCVD risk profile, atypical presentation for ACS, negative ECG changes, and an elevated troponin level believed to be due to the overall infectious presentation in support of myocarditis. The cardiac MRI for this patient, when interpreted in the clinical context of his presenting symptoms, seems to be more compatible with myocarditis than an infarct. On cardiac MRI, enhancement patterns in myocarditis generally exclude the subendocardium (excluding eosinophilic myocarditis). The subendocardium was not significantly enhanced on cardiac MRI, and rather the subepicardium was significantly affected in a patchy pattern.

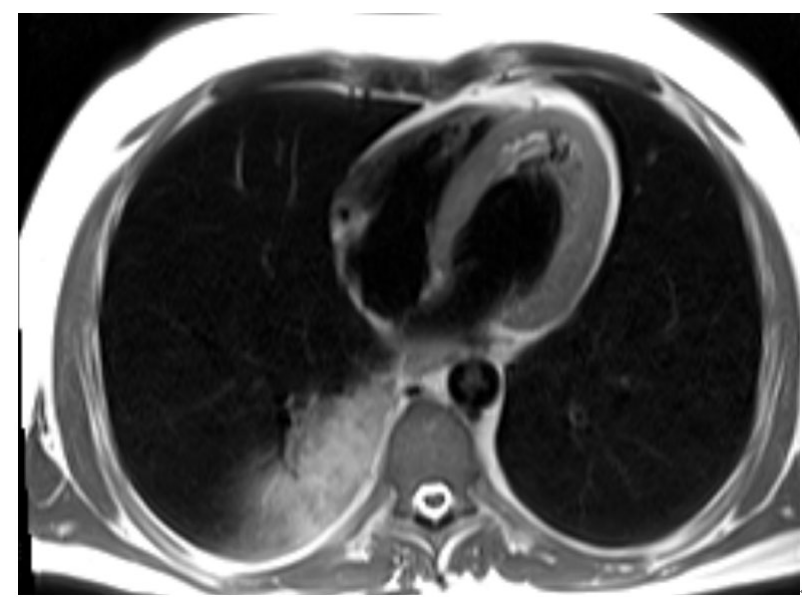

Figure 1. Axial view of the cardiac MRI depicting epicardial and mid-myocardial delayed gadolinium enhancement. There is also visualization of the right posterior lower lobe pneumonia

CXR demonstrated a right infrahilar opacification and medial right lower lung airspace consolidation concerning for pneumonia. The patient was started on oral levofloxacin 750 mg that was continued for one week. Troponin levels continued to trend down from an initial 12.47 to 3.53 over his four-day hospital stay. The patient was discharged in stable condition with outpatient cardiology follow-up within two weeks, after transition from ticagrelor to clopidogrel therapy on day four after a loading dose. Results of microbiology testing were all negative, except for coxsackie B1, B5, and B6, which were slightly positive with titers of $1: 32,1: 16$, 
and 1:32, respectively (normal $<1: 8$ ) and Rocky Mountain Spotted fever with titers of 1:64 (normal < 1:64).

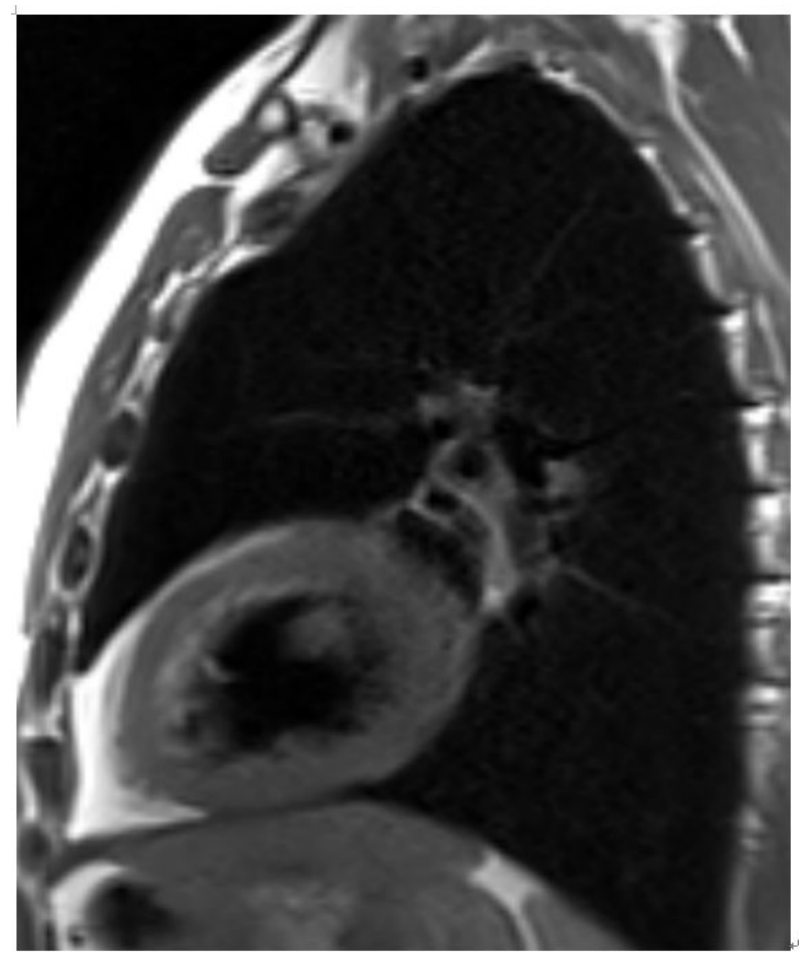

Figure 2. Scout view of the cardiac MRI depicting epicardial and mid-myocardial delayed gadolinium enhancement along the anterior wall

\section{Discussion}

Infectious myocarditis affects individuals worldwide. Beyond acute presentations, many hypotheses exist regarding long-term immunologic and inflammatory reactions or latent viral infections that later present at times of immunosuppression, therefore inducing chronic myocardial changes and damage. ${ }^{[2,5]}$ Guidelines on long-term monitoring and followup for chronic complications like cardiomyopathy in patients with self-limited myocarditis have not been established. Limited studies are available on independent risk factors associated with a poor prognosis, including baseline left ventricle function, NHYA functional heart classification at the time of presentation, and initial therapeutic management strategies. ${ }^{[6-8]}$ More studies are needed to identify risk factors for the development of chronic disease. Current methods to monitor for chronic cardiomyopathy include echocardiogram and newer cardiac MRI and PET, ${ }^{[9-11]}$ but timing of such interventions after initial diagnosis is unknown. ${ }^{[6]}$ Similarly, there are no guideline-based methods for treatment of chronic myocarditis, with research currently focusing on immunotherapy versus guideline-supported treatment of heart failure. ${ }^{[12]}$ As such, more studies identifying both prolonged imaging techniques and approach to treatment are required.

Despite the fact that cardiac MRI is far less invasive than myocardial biopsy in the diagnosis of myocarditis, the authors do not recommend routine testing for patients with suspected myocarditis at this time. The diagnosis of viral myocarditis can be obtained with a strong history and physical exam, and is adequately supported with the use of serum biomarkers, including ESR, CRP, and troponin, along with ECG, CXR, or transthoracic echocardiogram changes. While more invasive modalities like myocardial biopsy can be utilized in patients with an equivocal diagnosis, cardiac MRI offers an alternative modality to support the diagnosis of viral myocarditis in such cases that do not require an invasive procedure.

However, recent research of cardiac MRI in myocarditis may demonstrate a shift in the current diagnostic approach as more information is obtained about the lasting effects of myocarditis on the myocardium and subsequent cardiovascular outcomes. One such study identified that patients with myocarditis have persistent changes in late gadolinium enhancement on cardiac MRI, an important predictor of longtern cardiovascular outcomes. ${ }^{[13]}$ Therefore, while current guidelines do not support the routine use of cardiac MRI in the diagnosis and management of viral myocarditis, future studies may identify important features or risk factors on imaging that confer the need for routine follow-up to avoid cardiovascular complications in susceptible patients.

It can be argued that with the advent of new imaging modalities, the true incidence of viral myocarditis may be underestimated, as previously asymptomatic infections may be incidentally identified on cardiac MRI for other purposes. While this may be true and may play a small role in the increasing incidence of viral myocarditis, cardiac MRI is not routinely recommended for the diagnosis of viral myocarditis, and considerations of world climate change and increased international travel continue to be important in the rising incidence of this disease.

\section{Conclusion}

Standardized surveillance imaging and treatment for patients managed with acute infectious myocarditis is needed as incidence of myocarditis rises.

\section{CONFlicts OF InTEREST Disclosure}

The authors declare no conflicts of interest. 


\section{REFERENCES}

[1] Kang M, An J. Viral Myocarditis. In: StatPearls [Internet]. Treasure Island, FL: StatPearls Publishing; 2018. Available from: https: //www.ncbi.nlm.nih.gov/books/NBK459259/

[2] Arbustini E, Agozzino M, Favalli V, et al. Myocarditis. In: Hurst's The Heart. 14th ed. New York, NY: McGraw-Hill.

[3] $\mathrm{Ku} \mathrm{YH,} \mathrm{Yu} \mathrm{WL.} \mathrm{Fatal} \mathrm{Dengue} \mathrm{Myocarditis} \mathrm{despite} \mathrm{the} \mathrm{Use} \mathrm{of} \mathrm{Ex-}$ tracorporeal Membrane Oxygenation. Case Reports in Infectious Diseases. 2016; 2016: 1-2.

[4] Valdés O, Acosta B, Pi-ón A, et al. First report on fatal myocarditis associated with adenovirus infection in Cuba. Journal of Medical Virology. 2008; 80(10): 1756-61. PMid:18712847. https : //doi.org/10.1002/jmv. 21274

[5] Garmaroudi FS, Marchant D, Hendry R, et al. Coxsackievirus B3 replication and pathogenesis. Future Microbiology. 2015; 10(4): 62953. PMid:25865198. https://doi.org/10.2217/fmb.15.5

[6] Anzini M, Merlo M, Sabbadini G, et al. Long-Term Evolution and Prognostic Stratification of Biopsy-Proven Active Myocarditis. Circulation. 2013; 128(22): 2384-94. PMid:24084750. https : //doi.org/10.1161/CIRCULATIONAHA.113.003092

[7] Caforio AL, Calabrese F, Angelini A, et al. A prospective study of biopsy-proven myocarditis: prognostic relevance of clinical and aetiopathogenetic features at diagnosis. European Heart Journal. 2007 28(11): 1326-33. PMid:17493945. https://doi.org/10.1093/ eurheartj/ehm076
[8] Kindermann I, Kindermann M, Kandolf R, et al. Predictors of Outcome in Patients With Suspected Myocarditis. Circulation. 2008; 118(6): 639-48. PMid:18645053. https://doi.org/10.1161/CI RCULATIONAHA. 108.769489

[9] Bami K, Haddad T, Dick A, et al. Noninvasive imaging in acute myocarditis. Current Opinion in Cardiology. 2016; 31(2): 217-23. PMid:26731291. https ://doi.org/10.1097/HC0.0000000000 000265

[10] Cooper LT. The Changing Face of Cardiac Inflammation. Circulation: Heart Failure. 2017; 10(11). PMid:29158438. https : //doi .org/ 10.1161/CIRCHEARTFAILURE. 117.004528

[11] Grün S, Schumm J, Greulich S, et al. Long-Term Follow-Up of Biopsy-Proven Viral Myocarditis. Journal of the American College of Cardiology. 2012; 59(18): 1604-15. PMid:22365425. https : //doi.org/10.1016/j.jacc.2012.01.007

[12] Pollack A, Kontorovich AR, Fuster V, et al. Viral myocarditisdiagnosis, treatment options and current controversies. Nature Reviews Cardiology. 2015; 12(11): 670-80. PMid:26194549. https : //doi.org/10.1038/nrcardio.2015.108

[13] Berg J, Kottwitz J, Baltensperger N, et al. Cardiac Magnetic Resonance Imaging in Myocarditis Reveals Persistent Disease Activity Despite Normalization of Cardiac Enzymes and Inflammatory Parameters at 3-Month Follow-Up. Circulation: Heart Failure. 2017; 10(11). PMid:29158437. https://doi.org/10.1161/CIRCHEAR TFAILURE. 117.004262 